\title{
Português Língua Estrangeira/Adicional (PLE/PLA) e o Programa Idiomas sem Fronteiras ${ }^{1}$
}

\author{
Portugués Lengua Extranjera/Adicional (PLE/PLA) y el \\ Programa Idiomas sin Fronteras
}

\section{Portuguese as a Foreign/Additional Language (PFL/PAL) and the Languages Without Borders Program}

Denis Leandro Francisco ${ }^{2}$

\begin{abstract}
Resumo
Este artigo apresenta algumas das principais ações na área de Português Língua Estrangeira/Adicional (PLE/PLA) desenvolvidas no contexto de estruturação e implementação do Programa Idiomas sem Fronteiras (IsF), lançado pelo Ministério da Educação do Brasil (MEC) por intermédio da Secretaria de Educação Superior (SESu). São focalizadas as seguintes ações do IsF - Português: i. estudo-diagnóstico regionalizado sobre as condições da área de PLE/PLA nas 63 universidades públicas federais brasileiras; ii. avaliação de cursos e materiais didáticos na área de PLE/PLA disponibilizados em ambientes digitais; e iii. realização do Ciclo de Debates IsF - Português, ação de formação inicial na área de PLE/PLA desenvolvida integralmente a distância e direcionada aos representantes do programa nas 63 universidades públicas federais brasileiras.
\end{abstract}

Palavras-Chave: Português Língua Estrangeira/Adicional; PLE/PLA; Programa Idiomas sem Fronteiras; Políticas Linguísticas; Internacionalização.

\section{Resumen}

Este artículo presenta algunas de las acciones clave en el ámbito del Portugués como Lengua Extranjera/Adicional (PLE/PLA) desarrolladas en el contexto de la estructuración e implementación del Programa Idiomas sin Fronteras (IsF), puesto en marcha por el Ministerio de Educación de Brasil (MEC) a través de la Secretaría de Educación Superior (SESu). San focalizadas las siguientes acciones del IsF Portugués: i. estudio-diagnóstico regionalizado sobre las condiciones del área de PLE/PLA en las 63 universidades públicas federales brasileñas; ii. evaluación de cursos y materiales didácticos en el área de PLE/PLA disponibles en ambientes digitales; y iii. realización de un Ciclo de Debates, acción de formación inicial en el área de PLE/PLA realizada integralmente a distancia y dirigida a los representantes del programa en las 63 universidades públicas federales brasileñas.

Palabras claves: Portugués Lengua Extranjera/Adicional; PLE/PLA; Programa Idiomas sin Fronteras; Políticas lingüísticas; Internacionalización.

\begin{abstract}
This article presents some of the main actions in the area of Portuguese as a Foreign/Additional Language (PFL/PAL) developed in the context of structuring and implementation of the Languages Without Borders Program (IsF), launched by the Ministry of Education of Brazil (MEC) through the Secretariat of Higher
\end{abstract}

\footnotetext{
${ }^{1}$ Artigo apresentado durante o I Congresso Internacional Online de Estudos sobre Culturas - \#Culturas, realizado de forma online, em outubro de 2019.

2 Doutor em Letras; Universidade Federal de Lavras - UFLA; Lavras, Minas Gerais, Brasil; denisleandro@outlook.com
} 
Education (SESu). The following IsF - Portuguese actions are focused on: i. regionalized diagnostic study on the conditions of the PFL/PAL area in the 63 Brazilian federal public universities; ii. evaluation of courses and teaching materials in the area of PFL/PAL available in digital environments; and iii. holding the IsF - Portuguese Debate Cycle, an initial training course in the area of PLE/PLA developed entirely online.

Keywords: Portuguese as a Foreign/Additional Language; PFL/PAL; Languages without Borders Program; Language policies; Internationalization.

\section{Introdução}

Um dos principais desafios da educação superior brasileira é o seu processo de internacionalização, uma vez que tal processo envolve uma complexa definição de objetivos articulados com as demandas educacionais, com as diretrizes curriculares e com a legislação vigente, assim como com o Plano Nacional de Educação (PNE); envolve, ainda, o uso das tecnologias da informação e da comunicação, a formação de pessoal qualificado para a Educação a Distância e a definição de programas de estímulo visando à ampliação de intercâmbios internacionais, aspectos curriculares e organizacionais (ABREU-E-LIMA et al, 2017).

Entretanto, ainda que todas as premissas acima elencadas sejam atendidas, uma internacionalização efetiva não pode ser alcançada sem uma política linguística que possibilite, por um lado, que os estudantes e pesquisadores brasileiros se beneficiem da mobilidade acadêmica em países em que a língua oficial não é o português e, por outro lado, que os estudantes e pesquisadores estrangeiros falantes de outras línguas possam, por sua vez, ser inseridos nas universidades brasileiras.

É nesse panorama que surge o Programa Idiomas sem Fronteiras (IsF). O programa, lançado pelo Ministério da Educação (MEC) e instituído pela portaria n. ${ }^{\circ}$ 973, de 14 de novembro de $2014^{3}$, tem como principal objetivo "propiciar a formação e a capacitação em idiomas de estudantes, professores e corpo técnico-administrativo das Instituições de Educação Superior Públicas e Privadas - IES e de professores de idiomas da rede pública de educação básica, bem como a formação e a capacitação de estrangeiros em língua portuguesa" $\left(\right.$ Art. $1^{\circ}$ ). Segundo o inciso VI do artigo $2^{\circ}$ dessa portaria, o Programa Idiomas sem Fronteiras tem ainda como objetivos "fortalecer o ensino de idiomas no país, incluindo o da língua portuguesa, e, no exterior, o da língua portuguesa e da cultura brasileira".

Viabilizado graças ao trabalho desenvolvido em conjunto entre o Ministério da Educação (MEC), por intermédio da Secretaria de Educação Superior (SESu), a Coordenação de Aperfeiçoamento de Pessoal de Nível Superior (CAPES) e diversos outros parceiros

\footnotetext{
${ }^{3}$ Publicação no DOU n. ${ }^{\circ}$ 222, de 17.11.2014, Seção 1, página 11/12.
} 
internos e externos, o IsF apresenta um escopo abrangente, com duas frentes complementares: i. propiciar uma mudança estrutural no ensino de idiomas estrangeiros nas universidades do país, a fim de apoiar a experiência de internacionalização das instituições e dos estudantes brasileiros; e ii. fomentar a área de Português como Língua Estrangeira/Adicional (PLE/PLA), reconhecida como estratégica para o processo de internacionalização das Instituições de Ensino Superior (IES) brasileiras, promovendo a difusão da língua portuguesa no cenário internacional através de uma política própria para o ensino e a aprendizagem da língua portuguesa para falantes de outras línguas.

Como se pode perceber, o programa entende o ensino-aprendizagem de PLE/PLA como estratégico para o processo de internacionalização das universidades brasileiras, assim como para a promoção da imagem do Brasil no exterior. Consequentemente, o Idiomas sem Fronteiras - Português (IsF - Português) assume destacada importância no âmbito do Programa IsF, já que o fortalecimento e a divulgação da língua desempenham papel central no complexo processo de construção de uma política linguística para a internacionalização.

O conhecimento da realidade do ensino de PLE/PLA no âmbito nacional bem como o mapeamento desses cursos nas Instituições Federais de Ensino Superior (IFES) é, há muito, uma demanda importante da área acadêmica e, ao mesmo tempo, uma necessidade para a implementação de políticas públicas por parte do Governo Federal. Para que as ações do Ministério da Educação sejam eficazes quanto à elaboração de tais políticas visando à internacionalização dos estudantes brasileiros, é fundamental estruturar as universidades e dar condições para que consigam participar de forma ativa e prospectiva das ações de internacionalização propostas. É necessário, portanto, que o Ministério da Educação tenha condições de avaliar as necessidades das IES para direcionar esforços e planejar ações específicas que atendam a essas necessidades.

\section{Estudo-diagnóstico regionalizado sobre as condições da área de PLE/PLA nas 63 universidades públicas federais brasileiras}

Foi com o objetivo de facultar esse conhecimento da realidade do ensino de PLE/PLA no âmbito nacional que se realizou, entre setembro de 2014 e maio de 2015, período de estruturação do IsF - Português, a primeira ação a ser apresentada neste trabalho: um mapeamento regionalizado acerca das condições da área de PLE/PLA nas IFES brasileiras. Este estudo foi realizado no âmbito da Secretaria de Educação Superior do Ministério da Educação, com apoio da Organização das Nações Unidas para a Educação, a Ciência e a Cultura (UNESCO) (FRANCISCO, 2014). Pioneiro no que se refere à amplitude do 
diagnóstico que fornece da realidade da área de PLE/PLA no âmbito nacional, esse estudo certamente interessará a gestores de políticas linguísticas, mas também a professores e pesquisadores da área, a estudantes de graduação e de pós-graduação e a universidades estrangeiras interessadas em se informar sobre o panorama da área de PLE/PLA nas universidades brasileiras a fim de, por exemplo, estabelecer acordos de cooperação e mobilidade acadêmica com as nossas Instituições de Ensino Superior.

O mapeamento levantou aspectos institucionais e acadêmicos dos cursos de Letras PLE/PLA que a Educação Superior pode ofertar, como prevê o Art. 44 da LDB - Lei de Diretrizes e Bases da Educação Brasileira. São eles: cursos de graduação, de pós-graduação e de extensão. $\mathrm{O}$ estudo foi elaborado a partir da análise comparativa dos dados coletados por meio de pesquisa declaratória e de participação voluntária por parte dos representantes do IsF - Português nas IFES ou, no caso de tal representante não ter sido ainda nomeado pelo reitor, pelo responsável na Diretoria de Relações Internacionais ou, ainda, pelo representante do IsF - Inglês. Mediante coleta descentralizada de dados por meio de acesso on-line ao questionário disponibilizado através da ferramenta Survey Monkey, realizou-se um mapeamento detalhado da realidade dos cursos de língua portuguesa para estrangeiros, no âmbito da graduação, pósgraduação e extensão, traçando, assim, um diagnóstico do quadro de dificuldades enfrentadas pelas Instituições Públicas Federais de ensino superior das cinco regiões do Brasil.

$\mathrm{O}$ instrumento foi enviado a um conjunto de dez professores especialistas em PLE/PLA para ser submetido à leitura crítica e validação. Uma vez validado, ele foi reformulado para contemplar as sugestões de alteração feitas pela equipe de especialistas. A seguir, apresentamos um quadro comparativo com os 10 principais indicadores mapeados ao longo do primeiro ano de existência do Programa IsF - Português. 
RELACult - Revista Latino-Americana de Estudos em Cultura e Sociedade

Revista Latinoamericana de Estudios en Cultura y Sociedad | Latin American Journal of Studies in Culture and Society V. 06, ed. especial, mar., 2020, artigo n ${ }^{\circ} 1803$ | claec.org/relacult | e-ISSN: 2525-7870

\begin{tabular}{|c|c|c|c|c|c|c|c|c|c|c|c|c|}
\hline \multicolumn{13}{|c|}{ QUADRO-RESUMO PLE/PLA IFES } \\
\hline \multirow[b]{2}{*}{ 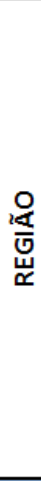 } & \multirow{2}{*}{\multicolumn{2}{|c|}{$\begin{array}{l}\text { 온 } \\
\underline{\underline{u}} \\
\underline{\underline{E}} \\
\underline{\underline{n}}\end{array}$}} & \multicolumn{10}{|c|}{ PERGUNTAS } \\
\hline & & & 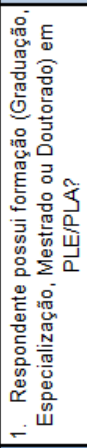 & 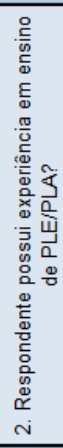 & 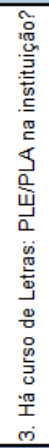 & 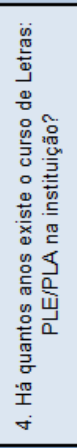 & 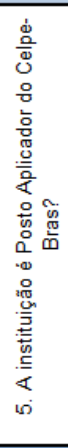 & 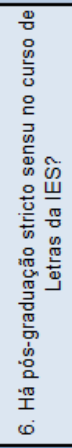 & 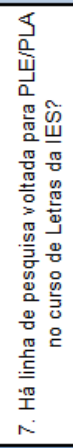 & 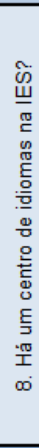 & 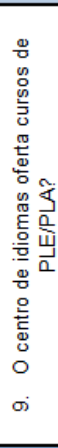 & 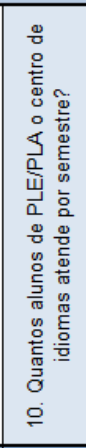 \\
\hline \multirow{11}{*}{ ș } & 1 & UFSC & & $x$ & & & $x$ & $x$ & $x$ & $x$ & $x$ & 50 \\
\hline & 2 & UFPEL & & $x$ & & & & $x$ & & $x$ & $\mathrm{x}$ & \\
\hline & 3 & UFSM & $\mathrm{x}$ & $x$ & & & $x$ & $x$ & & & & \\
\hline & 4 & UFCSPA & & $\mathrm{x}$ & & & $\mathrm{x}$ & & & $\mathrm{x}$ & $x$ & \\
\hline & 5 & FURG & & & $x$ & 1 & & & & $x$ & $x$ & \\
\hline & 6 & UFPR & & $x$ & & & $x$ & $x$ & $x$ & $x$ & $x$ & $301-400$ \\
\hline & 7 & UFFS & & & $x$ & 4 & & $x$ & $x$ & & & \\
\hline & 8 & UNILA & $\mathrm{x}$ & $x$ & $\mathrm{x}$ & 5 & $\mathrm{x}$ & $\mathrm{x}$ & & & & \\
\hline & 9 & UTFPR & & $x$ & & & & & & $x$ & $x$ & \\
\hline & 10 & UNIPAMPA & & $x$ & & & & $x$ & $x$ & $x$ & $x$ & \\
\hline & 11 & UFRGS & $x$ & $x$ & & & $x$ & $x$ & $x$ & $x$ & $x$ & $201-300$ \\
\hline \multirow{2}{*}{\multicolumn{2}{|c|}{ TOTAIS: }} & SIM & 3 & 9 & 3 & & 6 & 8 & 5 & 8 & 8 & \\
\hline & & NÃO & 8 & 2 & 8 & & 5 & 3 & 6 & 3 & 3 & \\
\hline \multirow{19}{*}{ 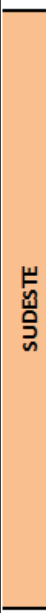 } & 12 & UNIRIO & & & & & & & & & & \\
\hline & 13 & UFU & & $x$ & & & & $x$ & & $x$ & & \\
\hline & 14 & UNIFESP & & & & & & $x$ & & & & \\
\hline & 15 & UFRRJ & & $x$ & & & & & & & & \\
\hline & 16 & UFLA & & $x$ & & & & & & $\mathrm{x}$ & & 51-100 \\
\hline & 17 & UFSCar & $x$ & $x$ & & & $x$ & $x$ & $x$ & & & 50 \\
\hline & 18 & UFVJM & & & & & & & & $\mathrm{x}$ & $x$ & 50 \\
\hline & 19 & UNIFEI & & $x$ & & & & & & & & 50 \\
\hline & 20 & UFRJ & $\mathrm{x}$ & $\mathrm{x}$ & $x$ & 30 & $x$ & $\mathrm{x}$ & $\mathrm{x}$ & $x$ & $x$ & $51-100$ \\
\hline & 21 & UFMG & $x$ & $x$ & & & $x$ & $x$ & $x$ & $x$ & $x$ & 51-100 \\
\hline & 22 & UFV & & $x$ & $x$ & 4 & & $x$ & & $x$ & $x$ & $51-100$ \\
\hline & 23 & UFF & $x$ & $x$ & & & $x$ & $x$ & $\mathrm{x}$ & $\mathrm{x}$ & $x$ & 50 \\
\hline & 24 & UFOP & $\mathrm{x}$ & $\mathrm{x}$ & & & & $\mathrm{x}$ & & $\mathrm{x}$ & $x$ & 50 \\
\hline & 25 & UFES & & & & & & $x$ & & $x$ & $x$ & 50 \\
\hline & 26 & UFJF & $x$ & $x$ & & & & $x$ & & $x$ & $x$ & 50 \\
\hline & 27 & UFTM & $\mathrm{x}$ & $x$ & & & & $x$ & & $x$ & & \\
\hline & 28 & UFSJ & & $x$ & & & & $x$ & & $x$ & $\mathrm{x}$ & 50 \\
\hline & 29 & UNIFAL & & & & & & $x$ & $x$ & & & \\
\hline & 30 & UFABC & & $\mathrm{x}$ & & & & & & & & \\
\hline \multirow{2}{*}{\multicolumn{2}{|c|}{ TOTAIS: }} & SIM & 7 & 14 & 2 & & 4 & 13 & 5 & 12 & 9 & \\
\hline & & NÃO & 12 & 5 & 17 & & 15 & 6 & 14 & 7 & 10 & \\
\hline \multirow{5}{*}{ 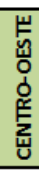 } & 31 & UFMS & & & & & & $x$ & & & $x$ & \\
\hline & 32 & UFMT & & $x$ & & & & $x$ & & $x$ & $x$ & $51-100$ \\
\hline & 33 & UFGD & & & & & & $x$ & & $x$ & & 50 (EAI) \\
\hline & 34 & UFG & $x$ & $x$ & & & & $x$ & & $\mathrm{x}$ & $x$ & 50 \\
\hline & 35 & UnB & $x$ & $x$ & $x$ & 16 & $x$ & $x$ & $x$ & $x$ & $x$ & 201-300 \\
\hline \multirow{2}{*}{\multicolumn{2}{|c|}{ TOTAIS: }} & SIM & 2 & 3 & 1 & & 1 & 5 & 1 & 4 & 4 & \\
\hline & & NÃO & 3 & 2 & 4 & & 4 & 0 & 4 & 1 & 1 & \\
\hline
\end{tabular}

Quadro 1 - Diagnóstico comparativo do contexto da área de PLE/PLA nas Instituições Federais de Ensino Superior brasileiras no ano de implantação do IsF - Português (regiões Sul, Sudeste e Centro-Oeste).

Fonte: FRANCISCO, D. L. Documento técnico: diagnóstico, avaliação, monitoramento e elaboração de propostas de programas SESu/MEC. Brasília: UNESCO/MEC/SESu, 2014. Projeto 914BRZ1144.6. Edital 010/2014 UNESCO/SESu. 
RELACult - Revista Latino-Americana de Estudos em Cultura e Sociedade

Revista Latinoamericana de Estudios en Cultura y Sociedad | Latin American Journal of Studies in Culture and Society V. 06, ed. especial, mar., 2020, artigo n ${ }^{\circ} 1803$ | claec.org/relacult | e-ISSN: 2525-7870

\begin{tabular}{|c|c|c|c|c|c|c|c|c|c|c|c|c|}
\hline \multicolumn{13}{|c|}{ QUADRO-RESUMO PLE/PLA IFES } \\
\hline \multirow[b]{2}{*}{ 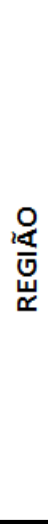 } & \multirow{2}{*}{\multicolumn{2}{|c|}{ 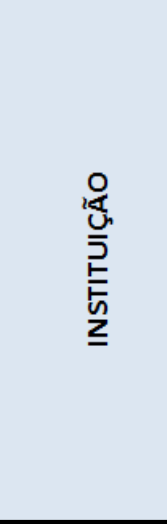 }} & \multicolumn{10}{|c|}{ PERGUNTAS } \\
\hline & & & 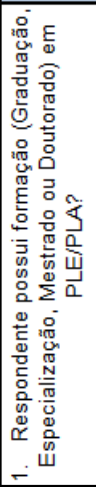 & 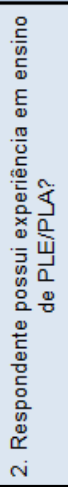 & 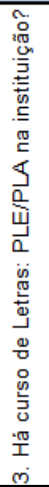 & 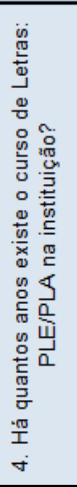 & 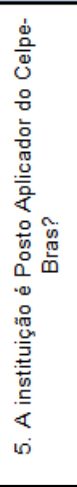 & 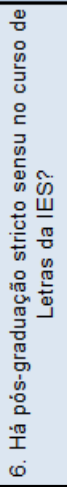 & 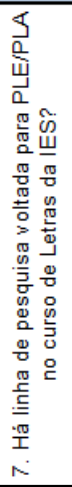 & 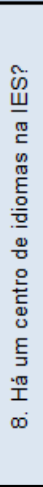 & 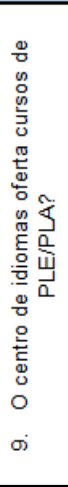 & 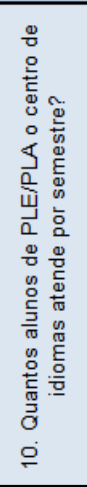 \\
\hline \multirow{10}{*}{$\begin{array}{l}\frac{\omega}{2} \\
\frac{0}{2} \\
2\end{array}$} & 36 & UFOPA & & & & & & $\mathrm{x}$ & & & & \\
\hline & 37 & UFT & & & & & & $x$ & & & & \\
\hline & 38 & UNIR & $x$ & $x$ & & & & $x$ & $x$ & & & \\
\hline & 39 & UFAC & & & & & & $x$ & & & & \\
\hline & 40 & UFAM & & $\mathrm{x}$ & & & $\mathrm{x}$ & $x$ & $x$ & $x$ & $x$ & 50 \\
\hline & 41 & UFPA & & $x$ & & & $x$ & $x$ & & $x$ & $x$ & 50 \\
\hline & 42 & UNIFAP & & $x$ & & & $x$ & & & $x$ & $\mathrm{x}$ & 50 \\
\hline & 43 & UFRR & & $x$ & & & $x$ & $x$ & & $x$ & $x$ & 50 \\
\hline & 44 & UFRA & & & & & & & & & & \\
\hline & 45 & UNIFESSPA & & & & & & $x$ & & & & \\
\hline \multirow{2}{*}{\multicolumn{2}{|c|}{ TOTAIS: }} & SIM & 1 & 5 & 0 & & 4 & 8 & 2 & 4 & 4 & \\
\hline & & NÃO & 9 & 5 & 10 & & 6 & 2 & 8 & 6 & 6 & \\
\hline \multirow{18}{*}{$\begin{array}{l}\frac{\omega}{y} \\
\text { 뭉 } \\
\frac{0}{2} \\
2\end{array}$} & 46 & UFRB & $x$ & $x$ & $x$ & 5 & & & & & & \\
\hline & 47 & UFCA & & & & & & & & $x$ & & \\
\hline & 48 & UFRN & $x$ & $x$ & & & & $x$ & $x$ & $x$ & $x$ & \\
\hline & 49 & UNIVASF & & & & & & & & $x$ & & \\
\hline & 50 & UFRPE & & & & & & & & $x$ & & \\
\hline & 51 & UFS & & & $x$ & 10 & $x$ & $x$ & $x$ & $x$ & $x$ & 50 \\
\hline & 52 & UFPI & & & & & & $x$ & & $x$ & & \\
\hline & 53 & UFAL & & & & & & $x$ & & $x$ & & \\
\hline & 54 & UNILAB & & $x$ & $x$ & 2 & & & & $x$ & $x$ & $51-100$ \\
\hline & 55 & UFCG & & & & & & $x$ & & $\mathrm{x}$ & & \\
\hline & 56 & UFSB & $x$ & $x$ & & & & & & $x$ & & \\
\hline & 57 & UFERSA & & & & & & & & & & \\
\hline & 58 & UFPB & & & & & $x$ & $x$ & $x$ & $x$ & & \\
\hline & 59 & UFBA & & $x$ & $x$ & 6 & $x$ & $x$ & $x$ & $x$ & $x$ & $101-200$ \\
\hline & 60 & UFC & & $x$ & & & & $x$ & & $x$ & & \\
\hline & 61 & UFPE & $x$ & $x$ & & & $x$ & $x$ & & $x$ & $x$ & $51-100$ \\
\hline & 62 & UFOB & & & & & & & & 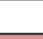 & & \\
\hline & 63 & UFMA & & & & & & $x$ & & $x$ & & \\
\hline \multirow{2}{*}{\multicolumn{2}{|c|}{ TOTAIS: }} & SIM & 4 & 7 & 4 & & 4 & 10 & 4 & 15 & 5 & \\
\hline & & NÃO & 14 & 11 & 14 & & 14 & 8 & 14 & 3 & 13 & \\
\hline \multicolumn{13}{|c|}{ PANORAMA NACIONAL - PLE/PLA } \\
\hline \multirow{2}{*}{\multicolumn{2}{|c|}{ TOTAIS: }} & SIM & 17 & 38 & 10 & & 19 & 44 & 17 & 43 & 30 & \\
\hline & & NÃO & 46 & 25 & 53 & & 44 & 19 & 46 & 20 & 33 & \\
\hline
\end{tabular}

Quadro 2 - Diagnóstico comparativo do contexto da área de PLE/PLA nas Instituições Federais de Ensino Superior brasileiras no ano de implantação do IsF - Português (regiões Norte e Nordeste).

Fonte: FRANCISCO, D. L. Documento técnico: diagnóstico, avaliação, monitoramento e elaboração de propostas de programas SESu/MEC. Brasília: UNESCO/MEC/SESu, 2014. Projeto 914BRZ1144.6. Edital 010/2014 UNESCO/SESu.

A partir dos dados fornecidos por esse estudo-diagnóstico, duas outras importantes ações puderam ser desenvolvidas: a avaliação de cursos e materiais didáticos na área de PLE/PLA disponibilizados em ambientes digitais e a realização do Ciclo de Debates IsF - 
RELACult - Revista Latino-Americana de Estudos em Cultura e Sociedade

Revista Latinoamericana de Estudios en Cultura y Sociedad | Latin American Journal of Studies in Culture and Society

V. 06, ed. especial, mar., 2020, artigo n ${ }^{\circ} 1803$ | claec.org/relacult | e-ISSN: 2525-7870

Português, ação de formação continuada realizada integralmente a distância e direcionada aos professores de PLE/PLA das 63 universidades públicas federais brasileiras.

\section{Avaliação de cursos e materiais didáticos na área de PLE/PLA disponibilizados em ambientes digitais}

A segunda ação realizou-se em três etapas: i. levantamento dos cursos on-line e materiais didáticos disponibilizados em ambientes digitais; ii. análise desses materiais com vistas a identificar quais poderiam ser incorporados ao portal do IsF - Português já na primeira etapa de estruturação do programa; e iii. elaboração de orientações sobre como utilizar esses materiais para autoestudo. A seguir, apresentamos uma amostra de materiais levantados, analisados e avaliados pelo Programa IsF - Português, bem como as orientações formuladas para auxiliar no uso autoinstrucional desses materiais.

Como posso aprender português?

VOCABULÁRIO

\section{NÍVEL DE PROFICIÊNCIA BÁSICO}

\section{BUSUU}

Habilidade avaliada: Vocabulário

Níveis de Proficiência: Básico, intermediário e avançado.

Público Alvo: Adolescentes e adultos nos contextos geral, acadêmico ou profissional.

Acesso: Gratuito, é necessário fazer cadastro.

Oferece feedback corretivo? Sim.

Pontos de destaque/Observações: O site oferece 5 cursos: Principiante A1, Básico A2 (estes dois foram aqui classificados como Básico), Intermediário B1, Intermediário Avançado B2 (este foi aqui classificado como Avançado) e um Curso para Viagens (aqui classificado como Básico). Esses cursos são divididos em lições a partir de temas de interação que vão da apresentação pessoal (A1) à discussão sobre lei ou religião (B2). O estudante tem a possibilidade de definir seus objetivos, diários, semanais, etc., e verificar sua progressão ao longo do curso. O site trabalha com um sistema de pontos (chamados de busuu-berries) que podem ser trocados por feedback de suas produções orais e escritas. Assim, para obter feedback de pessoas que se autodeclararam falantes nativos de português, o aprendiz é estimulado a dar feedback para produções de pessoas que estejam aprendendo a língua que o aprendiz declarou (no cadastro no site) como materna, formando uma espécie de rede social voltada para o aprendizado de línguas. É o único curso online de Português que oferece a opção de comunicação síncrona, via chat, com outros participantes do site. O Busuu promete ser uma opção alternativa ao método tradicional (gramatical); no entanto, as lições, em grande parte, estão baseadas em conteúdos gramaticais e de fixação de estruturas linguísticas. Os exercícios partem geralmente de uma palavra (ou expressão) que aparece contextualizada em um enunciado. Cabe ao estudante relacionar o texto oral ao escrito e, depois de certa introdução ao tema, resolver questões estruturais a partir de um diálogo artificialmente criado. Esses exercícios geralmente compreendem preenchimento de lacunas, organização de termos em um enunciado ou ordenação de falas em um diálogo imaginado. Há também exercícios de tradução de pequenos enunciados. Há conteúdos disponíveis somente para pagantes (contas Premium), mas não é obrigatório. Boa opção para conhecer pessoas, aprender vocabulário e praticar estruturas linguísticas.

Link: http://www.busuu.com/

Quadro 3 - Amostra de material didático PLE/PLA em ambiente digital.

Fonte: Fonte: Relatório Avaliativo de Sites e Materiais on-line do Programa IsF - Português.

Como posso aprender português?

COMPREENSÃO ORAL

\section{NÍVEL DE PROFICIÊNCIA INTERMEDIÁRIO}

\section{LIVEMOCHA}

Habilidade avaliada: Compreensão oral

Níveis de Proficiência: Básico e intermediário.

Público Alvo: Adolescentes e adultos. 
Acesso: Gratuito, é necessário fazer cadastro.

Oferece feedback corretivo? Sim.

Pontos de destaque/Observações:

Logo após o cadastro no site, o aprendiz seleciona uma imagem e uma frase que representem o nível de conhecimento do português que acredita ter. Há lições para os níveis: Fundamentos, Introdução, Iniciante (estes três foram aqui classificados como Básico) e Intermediário. Todas as lições seguem a mesma estrutura: Introdução, Vocabulário, Uso, Prática de Uso (com feedback automático), Ler-Escrever, Ler-Falar, Ouvir-Escrever e Ouvir-Falar. O site trabalha com um sistema de pontos que podem ser trocados por feedback de suas produções orais e escritas. Assim, para obter feedback de pessoas que se autodeclararam proficientes em português ou falantes nativos de português, o aprendiz é estimulado a dar feedback para produções de pessoas que estejam aprendendo uma das línguas que o aprendiz declarou (no cadastro no site) como materna ou proficiente, formando uma espécie de rede social voltada para o aprendizado de línguas. O aprendiz pode contar com uma ferramenta de tradução ao longo de todas as lições e tem total liberdade para traçar seu percurso de estudo, escolhendo a ordem das lições, inclusive passando de um nível de conhecimento para outro, sem nenhum tipo de obstáculo ou exigência. A compreensão oral perpassa todas as lições desde a Introdução. Podem ocorrer, na seção Prática de Uso, questões de compreensão oral, com feedback automático. As seções Ouvir-Escrever e Ouvir-Falar são voltadas especialmente para o exercício da compreensão oral: o aprendiz ouve a gravação e escreve ou grava sua resposta, obtendo feedback de participantes da rede. De modo geral, os diálogos foram construídos especialmente para o contexto de ensino-aprendizagem, não há ruídos, comuns nas interações cotidianas, e as falas são bastante pausadas.

Link: http://livemocha.com/

Quadro 4 - Amostra de material didático PLE/PLA em ambiente digital.

Fonte: Relatório Avaliativo de Sites e Materiais on-line do Programa IsF - Português.

\section{Ciclo de Debates IsF - Português}

A terceira ação, por sua vez, foi proposta com o objetivo principal de oferecer formação inicial na área de PLE/PLA àqueles representantes do IsF - Português que não tiveram contato prévio significativo com essa especialidade, de forma a: i. criar condições para a efetiva implementação do IsF - Português nas diferentes IFES que aderissem ao programa; ii. contribuir para a institucionalização e o fortalecimento da área de PLE/PLA nas IFES; e iii. estabelecer uma rede entre especialistas na área de PLE/PLA e profissionais que iriam atuar no âmbito do IsF - Português.

O Ciclo de Debates compreendeu uma carga-horária total de 120 horas. 105 dessas horas foram realizadas a distância, por meio de leituras, interações via webconferência e interações assíncronas via plataforma Moodle. Ao final do Ciclo, foi realizado um encontro presencial, em Brasília, durante 2 dias, reunindo todos os participantes, com carga-horária total de 15 horas. A parte a distância do Ciclo foi organizada em quatro módulos, a saber:

- Módulo I - Processos de institucionalização da área de PLE/PLA e políticas linguísticas de promoção internacional do português;

- Módulo II - Avaliação em PLE/PLA e o exame Celpe-Bras;

- Módulo III-Análise e produção de materiais didáticos de PLE/PLA;

- Módulo IV - Ensino-aprendizagem de PLE/PLA para fins, públicos e contextos específicos. 
Cada módulo compreendeu Oficinas Temáticas, organizadas em três momentos:

(i) leitura de um ou dois textos previamente indicados aos participantes e disponibilizados no Moodle (2 horas);

(ii) participação em webconferência com um palestrante convidado pelo IsF Português (1,5 horas). Durante a primeira hora, o palestrante discorreu sobre um dos temas elencados mais adiante; nos trinta minutos seguintes, os participantes fizeram perguntas ao convidado, que as respondeu na sequência;

(iii) participação em um fórum de discussão no Moodle, a partir de questões formuladas pelo palestrante da Oficina Temática (1,5 horas). Ao fim do fórum, o especialista sintetizou os principais pontos levantados, dando aos participantes as orientações que julgou pertinentes.

A equipe do IsF - Português convidou os diferentes palestrantes: docentes, pesquisadores ou pós-graduandos na área de PLE/PLA, preferencialmente com experiência significativa no tema da oficina de que estará encarregado. Coube a cada palestrante indicar um ou dois textos para leitura prévia por parte dos cursistas. Ele/a também se encarregou de abrir e finalizar o fórum de discussão.

Cada Oficina Temática teve, portanto, duração de 5 horas, distribuídas ao longo de duas semanas. Seus temas encontram-se especificados no quadro a seguir.

\begin{tabular}{|l|l|}
\hline Módulo & \multicolumn{1}{c|}{ Oficina Temática } \\
\hline I & Apresentação do Programa Idiomas sem Fronteiras - Português (IsF - Português) \\
\cline { 2 - 3 } (15 horas) & $\begin{array}{l}\text { Panorama contextual do ensino de PLE/PLA na contemporaneidade (no Brasil e no exterior): aspectos } \\
\text { contextuais, políticos, econômicos, sociais, metodológicos e operacionais }\end{array}$ \\
\cline { 2 - 3 } & Formação de professores de PLE / PLA \\
\hline \multirow{5}{*}{ II } & A Parte Escrita do Certificado de Proficiência em Língua Portuguesa para Estrangeiros (Celpe-Bras) \\
\cline { 2 - 3 } & A Parte Oral do Certificado de Proficiência em Língua Portuguesa para Estrangeiros (Celpe-Bras) \\
\cline { 2 - 3 } & Experiências inovadoras em avaliação de rendimento em PLE/PLA \\
\cline { 2 - 3 } & Níveis de proficiência em PLE/PLA e provas de nivelamento em PLE/PLA \\
\hline \multirow{5}{*}{ III horas) } & Perspectivas na análise e produção de materiais didáticos de PLE/PLA \\
\cline { 2 - 3 } & O lugar da cultura no ensino-aprendizagem de PLE/PLA \\
\cline { 2 - 3 } & Letramento crítico e multiletramentos no ensino-aprendizagem de PLE/PLA \\
\cline { 2 - 3 } & Compreensão oral e escrita no ensino-aprendizagem de PLE/PLA \\
\cline { 2 - 3 } & Produção escrita no ensino-aprendizagem de PLE/PLA \\
\cline { 2 - 3 } & Produção oral no ensino-aprendizagem de PLE/PLA \\
\cline { 2 - 3 } & A gramática e o vocabulário no ensino-aprendizagem de PLE/PLA \\
\hline \multirow{5}{*}{ IV horas) } & O ensino-aprendizagem de PLE/PLA em contexto de imersão \\
\cline { 2 - 3 } & O ensino-aprendizagem de PLE/PLA em ambientes digitais \\
\cline { 2 - 3 } & O ensino-aprendizagem de PLE/PLA via tandem \\
\cline { 2 - 3 } & O ensino-aprendizagem de PLE/PLA para fins acadêmicos \\
\cline { 2 - 3 } & O ensino-aprendizagem de PLE/PLA para falantes de espanhol \\
\cline { 2 - 3 } & O ensino-aprendizagem de PLE/PLA para falantes de línguas distantes \\
\cline { 2 - 3 } & $\begin{array}{l}\text { O ensino-aprendizagem de PLE/PLA como língua de herança e para cidadãos da Comunidade dos } \\
\text { Países de Língua Portuguesa }\end{array}$ \\
\hline
\end{tabular}

Quadro 5 - Relação das Oficinas Temáticas do Ciclo de Debates IsF - Português.

Fonte: Projeto Ciclo de Debates na área de Português Língua Estrangeira/Adicional para Representantes do Programa IsF - Português. 


\section{Conclusão}

Embora o papel estratégico da área de PLE/PLA para o processo de internacionalização das IES brasileiras e para a promoção e a difusão da língua portuguesa no cenário internacional seja reconhecido pelos órgãos de gestão da educação brasileira, conforme atestam documentos dedicados às políticas de internacionalização das nossas universidades públicas federais, uma das conclusões a que os dados obtidos por meio do estudo-diagnóstico realizado como primeira ação de estruturação do IsF - Português permitem chegar é que a área de PLE/PLA ainda carece de incentivos oficiais para a sua adequada institucionalização, principalmente quando se tem sua realidade comparada à de outros idiomas.

Este artigo mostra quão importantes são as ações desenvolvidas no contexto de estruturação do IsF - Português para o fortalecimento da área de PLE/PLA, especialmente aquela voltada para a formação inicial de professores e pesquisadores nessa área específica do ensino de línguas estrangeiras, pois essa iniciativa possibilitará a continuidade das demais ações propostas pelo programa ao longo da sua implementação. A ação Ciclo de Debates IsF - Português, nesse sentido, apresenta expressivas vantagens para a formação de professores da área, como, por exemplo, a alta capilaridade que uma ação realizada remotamente permite alcançar.

Ações como essa podem ter inúmeras aplicações para a formação docente (inicial ou continuada) na área de PLE/PLA, podendo ser estendidas para outras demandas da área, como a capacitação em serviço de professores que atuam nos Centros Culturais Brasileiros (CCB) e o treinamento de aplicadores do exame para obtenção do Certificado de Proficiência em Língua Portuguesa para Estrangeiros (Celpe-Bras), único exame brasileiro oficial para certificar proficiência em português para falantes de outras línguas maternas. O exame é aplicado anualmente em Postos Aplicadores ${ }^{4}$ no Brasil e no exterior pelo Instituto Nacional de Estudos e Pesquisas Educacionais Anísio Teixeira (Inep), com apoio do Ministério da Educação e em parceria com o Ministério das Relações Exteriores (MRE).

\footnotetext{
${ }^{4} \mathrm{Na}$ edição de maio de 2019, o Celpe-Bras foi aplicado em 106 postos em quase 40 países.
} 


\section{Referências}

ABREU-E-LIMA, D. M. et al. Perfil da área de língua inglesa nas universidades federais brasileiras. In: SARMENTO, S.; ABREU-E-LIMA, D. M.; MORAES FILHO, W. B. (Org.). Do Inglês sem Fronteiras ao Idiomas sem Fronteiras: a construção de uma política linguística para a internacionalização. Belo Horizonte: Editora UFMG, 2017.

FRANCISCO, D. L. Documento técnico: diagnóstico, avaliação, monitoramento e elaboração de propostas de programas SESu/MEC. Brasília: UNESCO/MEC/SESu, 2014. Projeto 914BRZ1144.6. Edital 010/2014 UNESCO/SESu.

INEP - Instituto Nacional de Estudos e Pesquisas Educacionais Anísio Teixeira. Inep aplica exame de proficiência em português em 106 postos no Brasil e exterior. Disponível em: http://portal.inep.gov.br/artigo/-/asset_publisher/B4AQV9zFY7Bv/content/inep-aplicaexame-de-proficiencia-em-portugues-em-106-postos-no-brasil-e-exterior/21206. Acesso em: 12 jun. 2019.

MEC - Ministério da Educação. Lei de Diretrizes e Bases da Educação Nacional (LDB) n ${ }^{\circ}$ 9.394/1996. Disponível em: http://www.planalto.gov.br/ccivil_03/Leis/L9394.htm. Acesso em: 22 mai. 2019.

MEC - Ministério da Educação. Portaria n ${ }^{\circ}$ 973, de 14 de novembro de 2014. Diário Oficial da União, Brasília, n. 222, Seção 1, p. 11, 17 nov. 2014. Disponível em: http://isf.mec.gov.br/images/pdf/novembro/Portaria_973_Idiomas_sem_Fronteiras.pdf.

Acesso em: 22 mai. 2019. 